\title{
Image-processing techniques in precisely measuring positions of Jupiter and its Galilean satellites
}

\author{
Q. Y. Peng ${ }^{1,2,5,6}$, Y. B. Han ${ }^{2}$, C. L. Zhang ${ }^{3}$, and Z. L. $\mathrm{Li}^{4,5}$ \\ 1 Department of Computer Science, Jinan University, Guangzhou 510632, PR China \\ 2 National Astronomical Observatories, CAS, Beijing 100012, PR China \\ 3 Department of Mathematics, Jinan University, Guangzhou 510632, PR China \\ 4 Yunnan Observatory, National Astronomical Observatories, CAS, Kunming 650011, PR China \\ 5 Joint Laboratory for Optical Astronomy, CAS, Kunming 650011, PR China \\ ${ }^{6}$ Center of Astrophysics, Guangzhou University, Guangzhou 510400, PR China
}

Received 2 July 2002/ Accepted 20 December 2002

\begin{abstract}
Two image-processing techniques are developed to measure precisely the positions of Jupiter and its Galilean satellites. One is used to detect the edge of Jupiter's CCD image and fit it with an ellipse, thereby obtaining the planetary center. The other is used to remove the positional effect of Jupiter's scattering light that has on the measurement of a close satellite. 133 frames of CCD images are measured by using these techniques, and these measured positions of Galilean satellites are compared to the ones computed with the ephemerides of Jacobson's JUP204 and Arlot's G5. Preliminary analysis of the data as Jupiter-satellite or inter-satellite positions shows that a standard error (i.e. internal precision) as small as 0.03 arcsec can be reached in right ascension and declination. It is believed that these techniques would be useful for astrometric and photometric CCD observations of Galilean satellites, especially for CCD observations in the forthcoming mutual phenomena of Galilean satellites.
\end{abstract}

Key words. techniques: image processing - planets and satellites: individual: Jupiter - astrometry

\section{Introduction}

Optical positions of the outer planets (Jupiter-Pluto) are important to improve and maintain their ephemerides since modern methods of observation (radar ranging and interferometer) are not effective at the distances involved (Standish et al. 1995; Standish 1998). In particular, these data are needed to plan and support spacecraft missions to the outer solar system (Cassini, Europa Orbiter, and Pluto-Kuiper Express).

In history, astrometric positions of the outer planets (Jupiter and Saturn) were not well determined by the classical observational methods. The external mean error of such observations varied from 200 to 500 mas (Standish 1995; Pascu \& Schmidt 1990). The largest systematic error was called "phase effect" and due to an augmentation of the geometric phase defect on the planets which was difficult to account for (Fienga 1998). At present, the positional observations of the outer planets (Jupiter and Saturn) have to be made first by the measurement of their satellites, and then derived by the comparison of satellites' measured positions and theoretical positions (for example, Rapaport et al. 2002; Stone 2001; Stone \& Harris 2000; Fienga 1998).

Send offprint requests to: Q. Y. Peng, e-mail: pengqy@public.guangzhou.gd.cn
In astrometry of some natural satellites, the precise measurement of the center of their primary planet is decisive. Because in some cases, to accomplish the removal of the background gradient produced by the over-exposed image of the planet, the planetary image is supposed to be symmetric relative to the two axes of a particular reference frame, both of which contain the planet's center. As we have known, the technique of the removal of the background gradient has been used in the reduction of some outer planets' satellites, such as Jovial, Saturnian and Uranian satellites (Veiga \& Martins 1995; Stone \& Harris 2000; Peng et al. 2002 - hereafter, called Paper I).

Nevertheless, the direct measurement for the outer planets (such as Jupiter, Uranus and Neptune) with good precision has not been made yet.

On the other hand, their geometric centers or geometric radii can be determined precisely for some celestial bodies with big disks. For example, the most recent observations (Penna et al. 2002) show that the solar radius can be measured with the precision of 0.18 arcsec using CCD Astrolabe. Imagining the huge geometric size of the solar surface, we think the measurement with so good precision must have its measurable base. Besides, Pascu \& Schmidt (1990) determined positions of Saturn by fitting 12 points around its ring obtained by manual setting with an ellipse. The internal precision due to the 


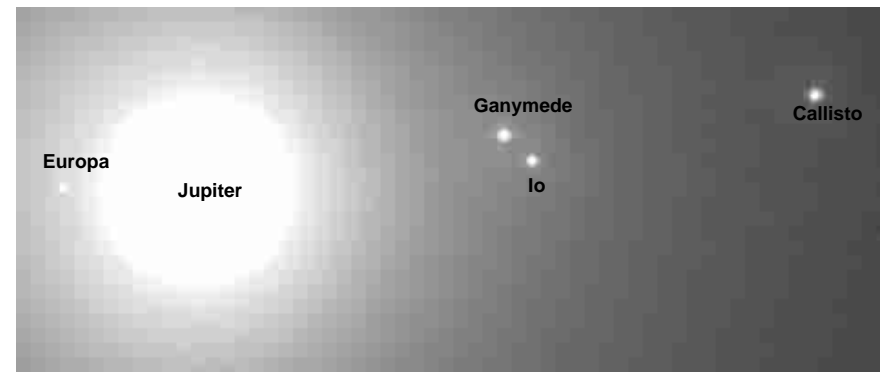

Fig. 1. A typical CCD image for the Jupiter and its Galilean satellites. The exposure time for this image is $2 \mathrm{~s}$.

measurement of this planet is as good as 0.10 arcsec. These methods have a common base, i.e., the edge of the celestial body with a big disk has a precise and measurable base.

Based on this idea, an elliptic fit to the edge of Jupiter on its CCD image is tried to determine its geometric center in the present paper. The positional precision is tested by many CCD images we have obtained. The results show that as good as $0.03 \mathrm{arcsec}$ of the internal precision can be reached. The remaining parts for this paper are arranged as follows: Sect. 2 gives the descriptions of our observations and instrumentation used; in Sect. 3, the positional measurement of Jupiter and its Galilean satellites is described in detail; Sect. 4 presents the calibration for the CCD field of view; Sect. 5 deals with results and analyses; the last Section will be the conclusion remarks.

\section{Instrumentation and observations}

On the night of Jan. 4, 2002 (the opposition of Jupiter is on Jan. 1, 2002), 133 frames of CCD images in total were obtained with the 1-meter telescope at the Yunnan Observatory. While observing, Johnson B-type filter was used. Besides, the exposure times of 1, 2 and 3 second(s) were adopted alternately (numbers of frames of CCD images are 44, 46 and 43, respectively for the exposure time of 1, 2 and 3 second(s)). In theory, we should have used Johnson I-type filter for Jupiter, a methane atmosphere planet. In fact, we had tried to use Johnson I-type filter at the beginning of the observation. But the overwhelming brightness of Jupiter always caused over-saturated within less than $1 \mathrm{~s}$ of exposure time. In order to obtain good quality images of both Jupiter and its Galilean satellites, we choose the Johnson B-type filter to decrease the brightness of Jupiter. Besides, the use of B-type filter allowed us to increase exposure time so that relative positions between any two satellites as well as Jupiter and a satellite have quite good precision. Although the used telescope was not specially made for astrometry, some publications (for example, Tang et al. 2000; Tang et al. 2001; Tang et al. 2002 and Paper I) have shown that it allows us to obtain good information on astrometry. For the detailed specifications of the telescope and CCD chip the reader may refer to Paper I. Figure 1 is a typical CCD image for the Jupiter and its Galilean satellites. As we have seen, Europa is the nearest satellite to the Jupiter, and Callisto is the farthest one.

\section{Positional measurement for the Jupiter and its Galilean satellites}

\subsection{Positional measurement for the Jupiter}

For not over-saturated CCD images of Jupiter, the following steps are taken to measure the position of the geometric center of Jupiter.

Step 1 . We choose a suitable rectangle that encloses a subimage with each side approximately twice as size as the diameter of visual surface of Jupiter (see Fig. 3a). After searching all pixels in the rectangle, we can find the maximum gray level $M$ and minimum one $m$.

Step 2. The sub-image is turned to a binary image after suitably thresholding. In order to obtain a precise position for the geometric center of Jupiter, an obvious prerequisite is to guarantee the measured position to be nearly constant when the same image is measured iteratively. That is to say, the measured position should hardly have relations to the size of the chosen rectangle and the tiny variation of the chosen threshold. Therefore, we have to search an optimal threshold by experiments. We assume a threshold in the form of

$V=m+(M-m)(\alpha+\beta / 300)$.

In this formula $\alpha$ is a small positive value ( 0.10 is adopted here) and $\beta$ is a factor varying in the range of $(0,1, \ldots, 200)$. The design of $\beta$ is to show a subtle graduation of Jupiter's geometric center measured in the following Step 4 and to search an optimal threshold. On the other hand, the design of a non-zero $\alpha$ is to guarantee a round figure on the whole for any binary image of Jupiter after thresholding. Otherwise (i.e. $\alpha=0$ ), the binary image of Jupiter would become a rectangle when $\beta=0$. Obviously, the execution of the ellipse-fit procedure in the following Step 4 would fail! Therefore, a non-zero value for $\alpha$ is indispensable when $\beta$ begins at 0 . Incidentally, the adopted 0.10 for $\alpha$ is obtained by our experiments.

Step 3. An edge of Jupiter is detected. Since a binary image for the Jupiter is not convenient to fit with a regular curve, a four-neighborhood mask (see Appendix A) is used to detect precisely its edge. Figure $3 \mathrm{~b}$ shows us an edge of Jupiter.

Step 4. Fit the edge with an ellipse by a least square and obtain its geometric center $(x, y)$.

After having obtained a series of centric positions of Jupiter corresponding to different $\beta$ factors, we have the following Fig. 2, which draws a figure with $\beta$ as its horizontal axis and with $x$ (or $y$ ) as its vertical axis for a CCD image obtained in a given exposure time. Since we have 3 different types of exposure times, Fig. 2 gives us 6 sub-figures. They allow us to choose a suitable $\beta$ factor for each type of exposure time. We find that a very good stability for the measured center $(x, y)$ in a large range of $\beta$ factors is shown for the image with a longest exposure time of $3 \mathrm{~s}$ (in the case of " $3 \mathrm{~s}$ exp-time" in Fig. 2), and also a quite good stability for the image with a median exposure time of $2 \mathrm{~s}$ (in the case of "2 s exp-time" in Fig. 2), but poor one for the image with a shortest exposure time of $1 \mathrm{~s}$ (in the case of "1 s exp-time" in Fig. 2). This phenomenon can be understood, since a shorter exposure time makes a less stable stellar image resulting from an abnormal atmosphere. In addition, irregular markings on the surface of Jupiter also make, 
Stability of positional measurement of Jupiter
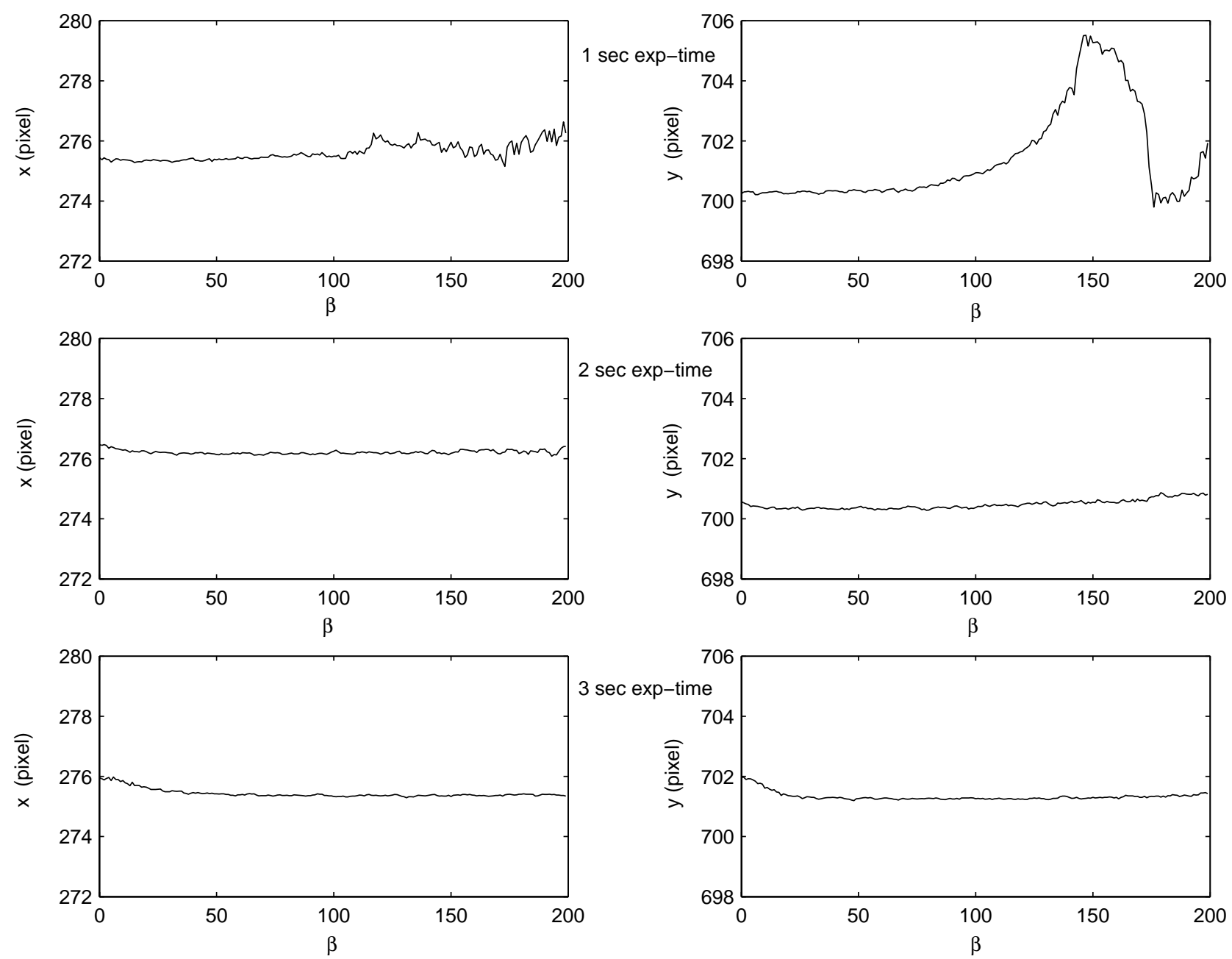

Fig. 2. The relationships between the raw pixel geometric center $(x, y)$ (unit: pixel) of Jupiter and different $\beta$ factors are given based on 3 CCD images obtained continuously with different exposure times. The left figures give the relationships between $x$ and $\beta$ and the right ones between $y$ and $\beta$. Abscissas are the range of $\beta$ factors.

sometimes greatly, this contribution (see the case of " $1 \mathrm{~s}$ exptime" in Fig. 2, when $\beta$ is greater than 100). Even so, there still exists a small range for $\beta$ that keeps some good stability, such as $\beta \in(0,70)$ corresponding to both coordinates. For a conservative estimation, $\beta \in(0,30),(30,100)$ and $(50,200)$ can be chosen for the images with 1,2 , and 3 second(s) of exposure time, respectively, in both coordinates. In our image-processing, the optimal $\beta \mathrm{s}$ of 15, 50 and 75 are adopted for the CCD images with 1, 2 and 3 second(s) of exposure time, respectively, in both coordinates.

\subsection{Positional measurement for the Galilean satellites}

As we mentioned in Sect. 1, a satellite, especially for the one near its primary planet (such as Jupiter, Saturn and Uranus), the removal of its halo is important. Here, the removal of halo of Jupiter is done in the same manner as the one Veiga \& Martins (1995) used for their processing for Uranian satellites. Figure 4 displays an image before and after the halo-removal for the faint satellite, Europa (left to the Jupiter). Here, if $\left(x_{0}, y_{0}\right)$ are the coordinates of the center of Jupiter, we subtract from every pixel $\left(x^{\prime}, y^{\prime}\right)$ in the region of the satellite the gray level of the pixel $\left(x^{\prime}, 2 y_{0}-y^{\prime}\right)$ or $\left(2 x_{0}-x^{\prime}, y^{\prime}\right)$ if $x$ or $y$, respectively, is the axis of symmetry. After that, a modified moment method is used to measure the raw-pixel position of a satellite. According to Stone (1989), the modified moment that suppresses the sky background below a prechosen threshold level is the best centering method when the sky-background level is significant. A further modified version of this method was made by Ji \& Wang (1996). The new modified moment method (still called modified moment method later on) has successfully been used in our previous measurement for Saturnian satellites (Paper I). In order to show the halo-removal effect, the same measurement with the modified moment method before and after the halo-removal has been performed. Figure 5 shows systematic shifts for Io, Europa, Ganymede and Callisto based on the difference of raw pixel coordinates between halo-exitance and halo-removal for the same satellite. In Fig. 5, the independent variable along the horizontal axis is the distance between the geometric center of Jupiter and a satellite. The following 


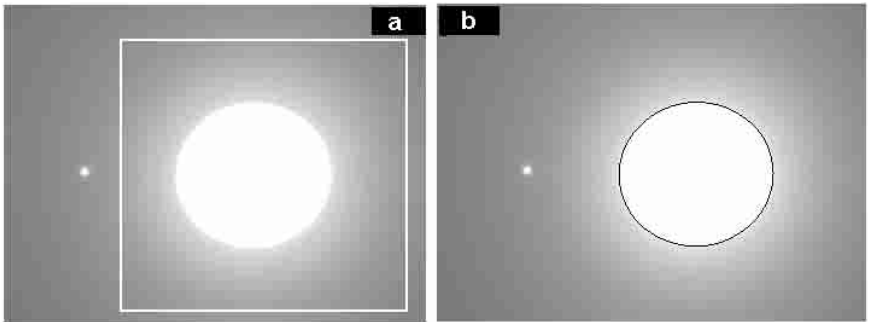

Fig. 3. Image processing for measuring of the geometric center of Jupiter. a) A rectangle enclosing the Jupiter in an image. b) An edge after the edge-detection.

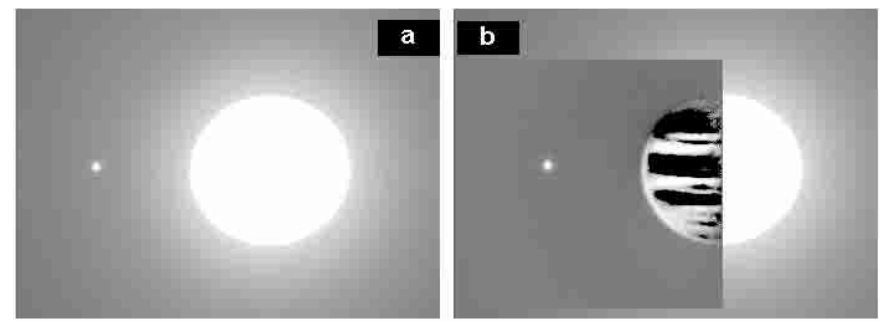

Fig. 4. The halo removal for the satellite Europa. a) An image before image processing. b) The same image after processing.

phenomena are found from Fig. 5. First, greater shifts exist in $x$ coordinate axis than that along $y$ coordinate axis for the same exposure time. This is easy to understand since the four satellites almost locate in $x$ coordinate on a CCD image (i.e. in the horizontal direction, also see Fig. 1). Second, an obvious greater shift is displayed for a shorter distance between a satellite and the Jupiter. For Io and Callisto, shifts along both axes are very small since their long distances from the Jupiter. Third, a longer exposure time results in a greater shift, Europa is the most obvious example. At last, a longer exposure time produces a greater dispersion for a mean systematic shift. Europa is also the most obvious example in this case. Incidently, the opposite sign of the shifts in $x$ and $y$ coordinates between Europa and the others results from their different positions. Europa is left to the Jupiter, but the others are right to the Jupiter. As we know, the center of any satellite is shifted towards the planetary center when the halo is not removed.

\section{Calibration for the CCD field of view}

We adopt two theories, G5 (Arlot 1982) and the newest JUP204 (Jacobson 2002), for the four Galilean satellites. In order to compute equatorial coordinates for the satellites, we also use JPL DE405 (Standish 1998) for the Jupiter. While calibrating the CCD field of view, we calculate topocentric positions for the Jupiter and Galilean satellites. Besides, both atmospheric refractions and the central projection (projection of the celestial sphere on the tangential plane of the focal point) are also taken into account. However, no phase effect is corrected for the observations of Galilean satellites since they are made very close to the opposition.

As well known, a small field of view of a CCD $(6 ! 4 \times 6 ! 4)$ has no enough reference stars chosen for calibrating the CCD device. We prefer to use a specific technique ("Brighter Moon
Table 1. The calibration parameters for the scale and orientation of CCD chip are computed by the JUP204 and G5 ephemerides respectively. $N$ gives the number of frames of CCD images.

\begin{tabular}{cccc}
\hline \hline $\begin{array}{c}\text { JUP204 } \\
\text { Subset }\end{array}$ & $N$ & $\begin{array}{c}\text { Scale } \\
\text { (arcsec/pixel) }\end{array}$ & $\begin{array}{c}\text { Orientation } \\
\text { (degree) }\end{array}$ \\
\hline 1 s exp-time & 43 & 0.373633 & 179.527 \\
2 s exp-time & 46 & 0.373631 & 179.529 \\
3 s exp-time & 44 & 0.373630 & 179.528 \\
\hline Total & 133 & 0.373631 & 179.528 \\
\hline G5 & $N$ & Scale & Orientation \\
Subset & & (degrecec) \\
\hline 1 s exp-time & 43 & 0.373657 & 179.521 \\
2 s exp-time & 46 & 0.373655 & 179.522 \\
3 s exp-time & 44 & 0.373654 & 179.522 \\
\hline Total & 133 & 0.373655 & 179.522 \\
\hline
\end{tabular}

Calibration" method), which has been used in the previous works (Harper et al. 1997; Shen et al. 2001; Vienne et al. 2001; and Paper I) for the calibration in our reduction. Strictly, the "Brighter Moon Calibration" method should only be used to obtain observations of inner faint satellites whose observational errors are large enough not to be affected by systematic errors in the scale and orientation caused by errors in the ephemerides of the bright satellites. Here, this method is used only to test our image-processing techniques and to assess the internal precision.

Table 1 gives results for the CCD calibration derived by using the four Galilean satellites. We see almost the same parameters for different exp-time subsets when the same ephemeris is used. Besides, for the same exp-time subset a very small difference exists when JUP204 and G5 are used respectively. The scales and orientations solved from all 133 frames of CCD images are adopted in the following reduction.

\section{Results and analyses}

If the scale and orientation of CCD chip are adopted, we can calculate conveniently the mean $(\mathrm{O}-\mathrm{C}) \mathrm{s}$ (Observed minus Computed) and standard errors of the observations in form of inter-satellite or Jupiter-satellite $\Delta \alpha \cos \delta$ and $\Delta \delta$ measures (see Vienne et al. 2001 and Paper I for detailed reference). In our implement, Ganymede has been chosen as the reference satellite since its near-center position in the field of view. Table 2 shows the mean $(\mathrm{O}-\mathrm{C}) \mathrm{s}$ and their standard errors in right ascension and declination of Io, Europa, Callisto and Jupiter. As shown in Table 2, the mean (O-C)s for each satellite and Jupiter are quite small when JUP204 is referred. Moreover, a very good agreement among the mean $(\mathrm{O}-\mathrm{C})$ s for $\mathrm{CCD}$ images obtained with different exposure time can be seen when the same ephemeris is referred. But slightly different standard errors when different exposure time is used mean that a longer exposure time produces better precision. We also note that almost all mean (O-C)s from G5 are greater than those from JUP204. The results suggest that JUP204 might be superior to G5 though one night observations cannot draw this conclusion. However, the best precision is found for Io among the four satellites, especially in right ascension and when G5 is 


\section{Halo effect on positional measurement of Galilean satellites}
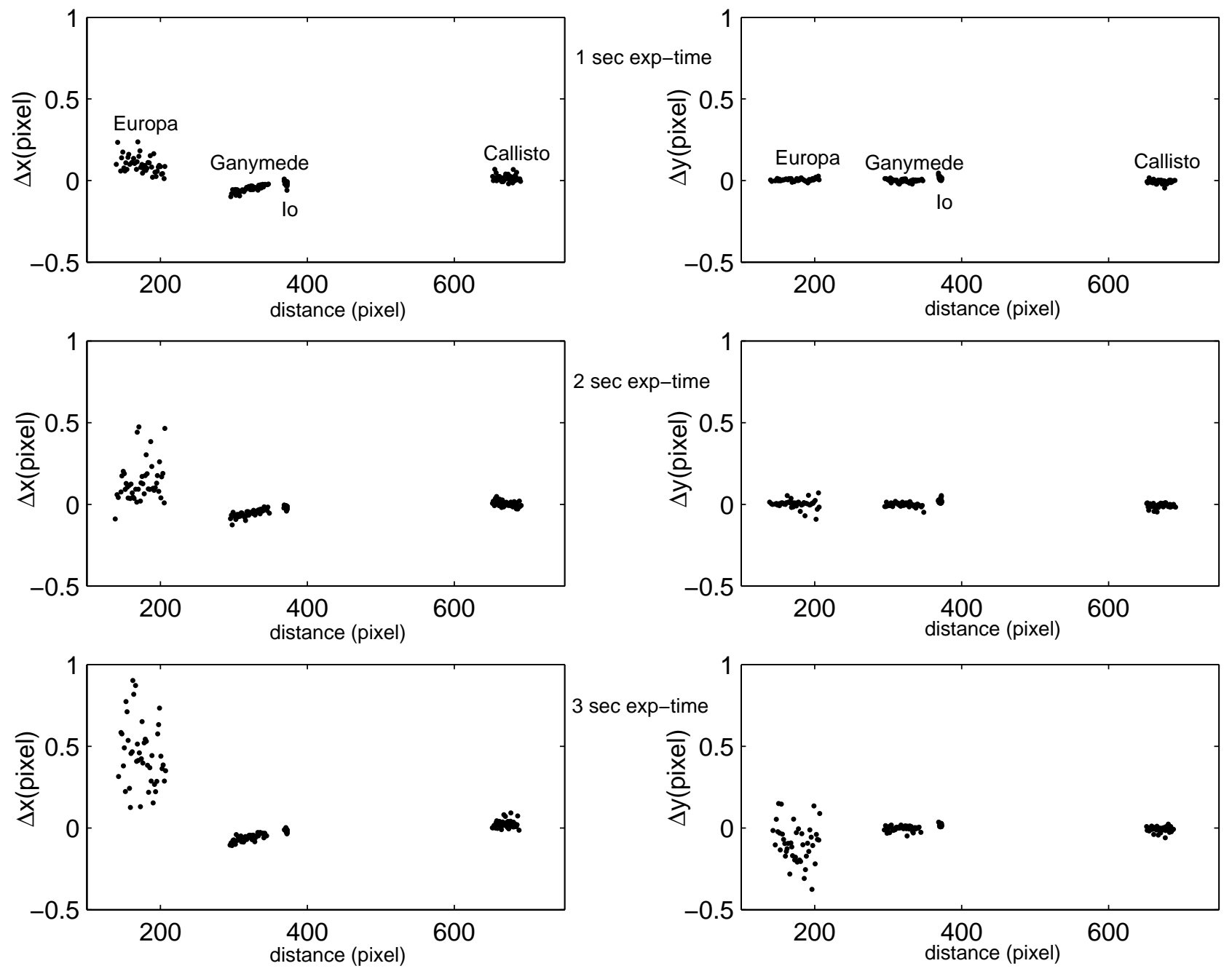

Fig. 5. Systematic shifts (unit: pixel) based on the difference of measured raw pixel coordinates before and after the halo-removal (i.e., in the sense of $\left.(\Delta x, \Delta y)=(x, y)_{\text {halo }}-(x, y)_{\text {no-halo }}\right)$. The left figures show the shifts along $x$-coordinate axis on an image and the right ones along $y$-coordinate axis. Abscissas give the distance (in pixel) between the geometric center of Jupiter and a satellite.

referred. It reveals a fact that a short separation between two satellites allows us to obtain very good positional precision. We believe that a short separation CCD observation would be very profitable to two Galilean satellites before and after their mutual phenomena-occultation. Better precision for Io when G5 is referred might be explained as a coincidence. In more detail, the variation of its light center might coincide with the variation of its prediction position of G5. In addition, the differences in mean (O-C)s for all satellites and Jupiter are usually small when two sets of calibration parameters are adopted respectively. The biggest difference is 23 mas for Europa in declination. This is understandable because of a quite great separation between this satellite and Ganymede. It suggests that a stricter calibration for the CCD field of view deserves to be investigated. At last, the appreciable mean $(\mathrm{O}-\mathrm{C})$ s even when the newest ephemeride JUP204 is used could be explained mainly as the difference in each satellite's positions between centerof-light relative to center-of-figure, since this difference may reach "the order of 75-100 km" (i.e., 25-33 mas for each satellite) according to Lieske (1998).

\section{Conclusion remarks}

Two image-processing techniques are developed to measure precisely the positions of Jupiter and its Galilean satellites. Tests based on 133 frames of CCD images we have obtained show that these techniques allow us to obtain as good as 0.03 arcsec precision for the positions of the center of Jupiter as well as those of the satellites. We are encouraged by the obtained internal precision. Investigators who are interested in the data processing are expected to check the observations using the original raw pixel positions and related records on request. It is believed that these techniques could be used in CCD astrometric observation and forthcoming mutual phenomena (occultation) observation. Further improvement in the edge-detection of Jupiter needs to be made in future when the planet is not near opposition. Perhaps, only the lamb on the sunward could 
Table 2. Statistics of the mean (O-C)s (Observed minus Computed) (unit: mas) of each satellite and Jupiter with respect to Ganymede. $N$ gives the number of observations. JI, JII and JIV represent Io, Europa and Callisto, respectively. Calibration parameters from JUP204 and G5, respectively, are adopted.

\begin{tabular}{|c|c|c|c|c|c|c|c|c|c|c|}
\hline & $\begin{array}{l}\text { Calibrated by } \\
\text { referred theory }\end{array}$ & JUP204 & & JUP204 & & & & G5 & & \\
\hline Subset & $N$ & object & $\begin{array}{c}\text { mean } \\
\Delta \alpha \cos \delta\end{array}$ & s.e. & $\begin{array}{c}\text { mean } \\
\Delta \delta\end{array}$ & s.e. & $\begin{array}{c}\text { mean } \\
\Delta \alpha \cos \delta\end{array}$ & s.e. & $\begin{array}{c}\text { mean } \\
\Delta \delta\end{array}$ & s.e. \\
\hline \multirow[t]{4}{*}{1 s exp-time } & 43 & JI & -47 & 13 & -2 & 11 & 50 & 9 & -4 & 12 \\
\hline & 43 & JII & 41 & 33 & -30 & 38 & 152 & 33 & -31 & 37 \\
\hline & 43 & JIV & 61 & 26 & -49 & 23 & 198 & 26 & -93 & 23 \\
\hline & 43 & Jupiter & -49 & 26 & -62 & 33 & 48 & 26 & -81 & 33 \\
\hline \multirow[t]{4}{*}{2 s exp-time } & 46 & JI & -46 & 13 & -3 & 12 & 50 & 8 & -4 & 13 \\
\hline & 46 & JII & 42 & 26 & -33 & 31 & 153 & 26 & -34 & 31 \\
\hline & 46 & JIV & 61 & 16 & -44 & 23 & 198 & 16 & -88 & 23 \\
\hline & 46 & Jupiter & -46 & 24 & -63 & 30 & 52 & 24 & -83 & 30 \\
\hline \multirow[t]{4}{*}{3 s exp-time } & 44 & JI & -46 & 12 & -3 & 10 & 52 & 6 & -4 & 10 \\
\hline & 44 & JII & 47 & 21 & -34 & 20 & 158 & 21 & -34 & 19 \\
\hline & 44 & JIV & 67 & 12 & -47 & 19 & 204 & 12 & -91 & 19 \\
\hline & 44 & Jupiter & -45 & 18 & -63 & 17 & 53 & 18 & -82 & 17 \\
\hline \multirow[t]{5}{*}{ Total } & 133 & JI & -46 & 13 & -2 & 11 & 51 & 8 & -4 & 11 \\
\hline & 133 & JII & 44 & 27 & -32 & 30 & 154 & 27 & -33 & 30 \\
\hline & 133 & JIV & 63 & 19 & -46 & 22 & 200 & 19 & -91 & 22 \\
\hline & 133 & Jupiter & -47 & 23 & -63 & 27 & 51 & 23 & -82 & 27 \\
\hline & $\begin{array}{l}\text { Calibrated by } \\
\text { referred theory }\end{array}$ & G5 & & JUP204 & & & & G5 & & \\
\hline Subset & $N$ & object & $\begin{array}{c}\text { mean } \\
\Delta \alpha \cos \delta\end{array}$ & s.e. & $\begin{array}{c}\text { mean } \\
\Delta \delta\end{array}$ & s.e. & $\begin{array}{c}\text { mean } \\
\Delta \alpha \cos \delta\end{array}$ & s.e. & $\begin{array}{c}\text { mean } \\
\Delta \delta\end{array}$ & $\overline{\text { s.e. }}$ \\
\hline \multirow[t]{4}{*}{$1 \mathrm{~s}$ exp-time } & 43 & JI & -49 & 14 & 0 & 11 & 48 & 9 & -2 & 11 \\
\hline & 43 & JII & 50 & 33 & -52 & 38 & 169 & 33 & -53 & 37 \\
\hline & 43 & JIV & 55 & 26 & -33 & 23 & 192 & 26 & -77 & 23 \\
\hline & 43 & Jupiter & -44 & 26 & -77 & 32 & 53 & 26 & -96 & 32 \\
\hline \multirow[t]{4}{*}{2 s exp-time } & 46 & JI & -49 & 13 & -1 & 12 & 48 & 8 & -2 & 12 \\
\hline & 46 & JII & 51 & 26 & -56 & 31 & 162 & 26 & -56 & 31 \\
\hline & 46 & JIV & 54 & 16 & -27 & 23 & 192 & 16 & -72 & 23 \\
\hline & 46 & Jupiter & -41 & 24 & -78 & 30 & 57 & 24 & -98 & 30 \\
\hline \multirow[t]{4}{*}{3 s exp-time } & 44 & JI & -48 & 12 & -1 & 9 & 49 & 7 & -3 & 10 \\
\hline & 44 & JII & 57 & 21 & -56 & 20 & 167 & 21 & -57 & 19 \\
\hline & 44 & JIV & 60 & 12 & -30 & 19 & 198 & 12 & -75 & 19 \\
\hline & 44 & Jupiter & -40 & 18 & -78 & 17 & 58 & 18 & -97 & 17 \\
\hline \multirow[t]{4}{*}{ Total } & 133 & JI & -49 & 13 & -1 & 11 & 48 & 8 & -2 & 11 \\
\hline & 133 & JII & 53 & 27 & -55 & 30 & 163 & 27 & -56 & 30 \\
\hline & 133 & JIV & 56 & 19 & -30 & 22 & 194 & 19 & -75 & 22 \\
\hline & 133 & Jupiter & -42 & 23 & -78 & 27 & 56 & 23 & -97 & 27 \\
\hline
\end{tabular}

be used to fit by an ellipse after the edge detection, since the edge on the terminator of Jupiter is not sharp enough at this time. Besides, in order to obtain high accuracy in astrometric positions of Jupiter and its Galilean satellites, we should be careful to use the "Brighter Moon Calibration" method and better calibration methods deserve to be investigated.

\section{Appendix A}

The algorithm of four-neighborhood mask for the edgedetection can be explained using the following $\mathrm{C}++$ codes. int source-image $[\mathrm{M}][\mathrm{N}]$;

$I^{*}$ a binary image before the edge-detection with a size of $\mathrm{M} \times \mathrm{N}$. In this image, if the gray level of a point in the binary image is 0 , it is meant that the point locates in the background.
On the contrary, if the gray level of a point is 1 , the point locates in the foreground. */

int object-image[M-1][N-1];

$/ *$ the binary image after source-image $[\mathrm{M}][\mathrm{N}]$ is processed by the edge-detection. $* /$

int $\mathrm{p}$; //any detected point

int $\mathrm{n}, \mathrm{s}, \mathrm{w}, \mathrm{e}$;

//four neighborhood points of the detected point $\mathrm{p}$ int $\mathrm{i}, \mathrm{j} ; / /$ loop variables

for $(\mathrm{i}=1 ; \mathrm{i} ; \mathrm{M}-1 ; \mathrm{i}++)$

$$
\begin{aligned}
& \text { for }\left(j=1 ; j_{i} N-1 ; j++\right) \\
& \quad p=\text { source-image }[\mathrm{i}][\mathrm{j}] \text {; } \\
& \quad \text { if }(\mathrm{p}==0) \\
& \quad \text { object-image }[\mathrm{i}][\mathrm{j}]=0 ;
\end{aligned}
$$


else\{

$$
\begin{aligned}
& n=\text { source-image }[\mathrm{i}-1][\mathrm{j}] \\
& \mathrm{s}=\text { source-image }[\mathrm{i}+1][\mathrm{j}] \\
& \mathrm{w}=\text { source-image }[\mathrm{i}][\mathrm{j}-1] \\
& \mathrm{e}=\text { source-image }[\mathrm{i}][\mathrm{j}+1] \\
& \text { int temp=n+s+w+e; } \\
& \text { if }(\text { temp==4) } \\
& \text { ebject-image }[\mathrm{i}][\mathrm{j}]=0 ;
\end{aligned}
$$$$
\text { object-image }[\mathrm{i}][\mathrm{j}]=1 \text {; }
$$

$/ *$ if temp equals 4 , the point $\mathrm{p}$ and its four neighborhood points are all in the foreground (i.e., the interior of the detected-edge). Therefore, only the exact edge points will be detected. */

$$
\text { \} }
$$

// end of the algorithm

Acknowledgements. We would like to thank the referee-Dr Dourneau G. for his valuable comments to improve the scientific quality of the paper. We are grateful to Prof Shen K.X. for giving us helpful suggests and doing some modifications on the original manuscript. We are also grateful to Dr Jacobson J.B. for his kind help for us to obtain correctly the newest ephemeride JUP204. It is a pleasure to thank Dr Vienne A., Dr Emelianov N.V. and Prof Zhang C.Z. for giving us helpful suggestions. This work was carried out with financial support partly from the National Natural Science Foundation of China (No 10273015), and partly from the College of Information Science and Technology, Jinan University.

\section{References}

Arlot, J. E. 1982, A\&A, 107, 305

Fienga, A. 1998, A\&A, 335, 1111

Harper, D., Murray, C. D., Beurle, K., et al. 1997, A\&AS, 121, 65

Ji, K. F., \& Wang, F. 1996, AcASn, 37, 85

Jacobson, J. B. 2002, JUP204-JPL satellite ephemeris

Lieske, J. H. 1998, A\&AS, 129, 205

Pascu, D., \& Schmidt, R. E. 1990, AJ, 99, 1974

Peng, Q. Y., Vienne, A., \& Shen, K. X. 2002, A\&A, 383, 296

Penna, J. L., Jilinski, E. G., Andrei, A. H., et al. 2002, A\&A, 384, 650

Rapaport, M., Teixeira, R., Campion, J. F. L., et al. 2002, A\&A, 383, 1054

Shen, K. X., Dourneau, G., Qiao, R. C., et al. 2001, A\&A, 367, 1061

Standish, E. M. Jr., Newhall, X. X., Williams, J. G., et al. 1995, JPL planetary and Lunar Ephemerides, DE403/LE403 (JPL Interoffice Memo.314.10-127) (Pasadena: JPL)

Standish, E. M. Jr. 1998, JPL planetary and Lunar Ephemerides, DE405/LE405 (JPL Interoffice Memo.312.F-98-048.0) (Pasadena: JPL)

Stone, R. C. 1989, AJ, 97, 1227

Stone, R. C., \& Harris, F. H. 2000, AJ, 119, 1985

Stone, R. C. 2001, AJ, 120, 2124

Tang, Z. H., Wang, S. H., \& Jin, W. J. 2000, MNRAS, 319, 717

Tang, Z. H., Wang, S. H., \& Jin, W. J. 2001, AJ, 121, 1199

Tang, Z. H., Wang, S. H., \& Jin, W. J. 2002, AJ, 123, 125

Veiga, C. H., \& Vieira Martins, R. 1995, A\&AS, 111, 387

Vienne, A., Thuillot, W., Veiga, C. H., et al. 2001, A\&A, 380, 727 